\title{
Giant Alkali-Metal-Induced Lattice Relaxation as the Driving Force of the Insulating Phase of Alkali-Metal/Si(111):B
}

\author{
L. Chaput, ${ }^{1}$ C. Tournier-Colletta, ${ }^{1}$ L. Cardenas, ${ }^{1}$ A. Tejeda, ${ }^{1,2}$ B. Kierren, ${ }^{1}$ D. Malterre, ${ }^{1}$ and Y. Fagot-Revurat ${ }^{1}$ \\ ${ }^{1}$ Institut Jean Lamour UMR 7198, Nancy Université/CNRS, B.P. 70239 F-54506 Vandouvre-lès-Nancy, France \\ ${ }^{2}$ Synchrotron SOLEIL, L'Orme des Merisiers, St Aubin, 91192 Gif sur Yvette, France \\ P. Le Fèvre, F. Bertran, and A. Taleb-Ibrahimi \\ Synchrotron SOLEIL, L'Orme des Merisiers, St Aubin, 91192 Gif sur Yvette, France
}

D. G. Trabada, J. Ortega, and F. Flores

Departamento de Física Téorica de la Materia Condensada, Universidad Autonóma de Madrid, Campus de Cantoblanco, 28049 Madrid, Spain

(Received 10 June 2011; published 28 October 2011)

\begin{abstract}
$A b$ initio density-functional theory calculations, photoemission spectroscopy (PES), scanning tunneling microscopy, and spectroscopy (STM, STS) have been used to solve the $2 \sqrt{3} \times 2 \sqrt{3} R 30$ surface reconstruction observed previously by LEED on $0.5 \mathrm{ML} \mathrm{K} / \mathrm{Si}: \mathrm{B}$. A large K-induced vertical lattice relaxation occurring only for $3 / 4$ of $\mathrm{Si}$ adatoms is shown to quantitatively explain both the chemical shift of $1.14 \mathrm{eV}$ and the ratio $1 / 3$ measured on the two distinct $\mathrm{B} 1 s$ core levels. A gap is observed between valence and conduction surface bands by ARPES and STS which is shown to have mainly a Si-B character. Finally, the calculated STM images agree with our experimental results. This work solves the controversy about the origin of the insulating ground state of alkali-metal/Si(111): $\mathrm{B}$ semiconducting interfaces which were believed previously to be related to many-body effects.
\end{abstract}

DOI: 10.1103/PhysRevLett.107.187603

PACS numbers: 79.60.Bm, 68.37.Ef, 71.15.Mb

Semiconducting surfaces being $\sqrt{3} \times \sqrt{3} R 30$ - reconstructed $(\sqrt{3}-$ in the following) exhibit $s p$-type surface dangling bond bands narrow enough to obtain $2 \mathrm{D}$ half-filled Mott insulators as early proposed in alkali-metal/Si(111):B $[1,2]$ and $\mathrm{SiC}(0001)$ [3]. Indeed, metallic surface states were obtained from density-functional theory (DFT) calculations $[4,5]$ whereas large gaps have been measured experimentally $[1,6]$. The Mott metal-insulator transition $[7,8]$ was proposed to be achieved in the low temperature $\sqrt{3}$ phases of $\mathrm{Sn} / \mathrm{Ge}(111)$ [9] and more recently in $\mathrm{Sn} / \mathrm{Si}(111)$ [10]. In addition, the triangular topology common to all these materials has even been predicted to exhibit magnetic properties originating from the frustrated triangular lattice [11-13] and superconductivity upon doping [14]. Nevertheless, surface reconstructions may enhance many-body effects as proposed for the precursive $3 \times 3$ phase of $\mathrm{Sn} / \mathrm{Ge}(111)$ [15] as well as reduce them by favoring insulating states such as the $\mathrm{Si}(111)-2 \times 1$ well accounted in band theory $[16,17]$. This evidences a complex interplay between lattice and electronic degrees of freedom at surface. In this context, a novel $2 \sqrt{3} \times 2 \sqrt{3} R 30$ surface reconstruction $(2 \sqrt{3}$ in the following) has been evidenced close to saturation in alkali-metal/Si(111):B [18]. Angle-resolved photoemission measurements reveal the $2 \sqrt{3}$ symmetry and an alkalimetal-dependent gap [19]. A novel interpretation in terms of a lattice-driven bipolaronic insulating ground state, instead of a Mott-Hubbard one, has been proposed showing a quantitative agreement with the angle-resolved photoemission spectroscopy (ARPES) spectra [18]. Contrary to former assumption, saturation coverage has been proved to be 0.5 monolayer leading to the possible alternation of empty and doubly occupied Si dangling bonds in agreement with the bipolaronic model on the hexagonal lattice $[19,20]$. Nevertheless, the surface reconstruction and the corresponding charge order have not been solved up to now. Core levels photoemission spectroscopy (CLPES) is known to be a powerful tool to investigate the charge distribution at surface and its combination with STM cartographies can be crucial to determine the local atomic pattern [21]. In this Letter, experimental techniques such as high resolution core levels and valence band PES, STM and STS (scanning tunneling spectroscopy) have been coupled to DFT calculations in order to determine the local atomic structure of the $2 \sqrt{3}$-surface reconstruction. It is found that a giant $\mathrm{K}$-induced modulation of the $\mathrm{B}$-Si adatom bonds is able to explain both the insulating ground state characterized by ARPES and STS as well as the charge transfer observed from B $1 s$ core levels leading to the main conclusion that many-body physics is not necessary to explain the strong insulating nature of alkali-metal/Si:B materials.

CLPES measurements were carried out at the Cassiopee beam line of the synchrotron SOLEIL. High resolution ARPES and STS data have been recorded in Nancy. The results of $a b$ initio calculations presented here have been obtained with the WIEN2K computer code. B-enriched 
$\operatorname{Si}(111)$ substrates $\left(\rho \approx 10^{-3} \Omega \mathrm{cm}\right)$ have been first prepared by several short annealing at $1450 \mathrm{~K}$ followed by a few hours heating at $1100 \mathrm{~K}$ to favor segregation of boron atoms at the surface and trigger the $\sqrt{3}$ - surface reconstruction. Then, the $\mathrm{K} / \mathrm{Si}: \mathrm{B}$ interface has been prepared by evaporation of alkali-metal atoms from a SAES getter with a deposition rate of 0.13 monolayer/mn in a vacuum better than $8 \times 10^{-10}$ mbar.

The evolution of LEED patterns and $\mathrm{K} 2 p$ core levels as a function of alkali-metal coverage has established the conservation of $\sqrt{3}$ pattern in the low coverage regime, followed by a $3 \times 3$ for intermediate coverage competing with a $2 \sqrt{3}$ at saturation [19]. This has been shown to be a common feature of alkali-metal/Si:B ultrathin films and, unlike previous considerations, it has been evidenced very recently that the saturation coverage corresponds to $\theta_{s}=$ 0.5 alkali-metal monolayer [20]. Previous CLPES measurements of the coverage dependence of B $1 s$ core levels have been shown to exhibit two distinct regimes: a single peak is observed for the Si:B substrate whereas a spectral weight transfer occurs through a second low binding energy contribution above half of the saturation coverage [22-25]. At saturation coverage, two well-defined B $1 s$ peaks are observed as presented in Fig. 1(a) for K/Si:B films, their respective area approaching the ratio $1 / 3$. A unique boron site (noticed $S_{5}$ ) characterizing the $\sqrt{3}$ reconstruction of the Si:B substrate is expected [26,27]. A global shift of $0.52 \mathrm{eV}$ of the B $1 s$ spectra toward higher binding energy upon $\mathrm{K}$ deposition has been also observed for both Si $2 p$ core levels and valence band spectra and has been interpreted as a band bending effect due to the formation of the alkali-metal/Si:B interface [25]. The occurrence of two B $1 s$ features has been previously demonstrated and can be now interpreted in the light of our recent work [19] as clear evidence that the $2 \sqrt{3}$ surface

(a)

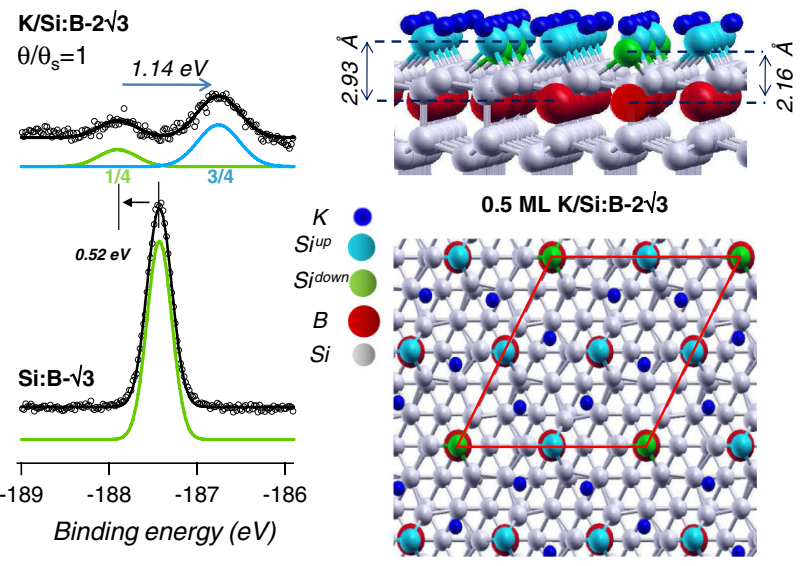

FIG. 1 (color online). (a) B $1 s$ core levels measured at $h \nu=$ $230 \mathrm{eV}$ on Si:B (down) and 0.5 ML K/Si:B $-2 \sqrt{3}$ (up); (b) top and side views of the $0.5 \mathrm{ML} \mathrm{K} / \mathrm{Si}: \mathrm{B}-2 \sqrt{3}$ structure obtained from DFT calculations. reconstruction exhibits two locally distinct $\mathrm{B}$ sites corresponding to $1 / 4$ and $3 / 4$ of boron atoms per unit cell. This should be a direct signature of the inhomogeneous electron doping of dangling bonds, i.e., the charge transfer induced by alkali-metal adsorption.

Therefore, starting both from the evidence of two distinct $\mathrm{B}$ sites and from a 0.5 monolayer saturation coverage corresponding to $6 \mathrm{~K}$ adatoms per reconstructed unit cell, we have performed DFT calculations to resolve the nature of the $2 \sqrt{3}$ surface reconstruction. We have explored possible ground state geometries for different coverage and qualitatively different geometries using an efficient localorbital molecular dynamics technique (FIREBALL) [28]. The 0.5 coverage was performed with the LAPW-DFT method implemented in the WIEN2K computer code. The PBE [29] approximation was used for the exchange correlation potential. We used muffin-tin radii of 1.83 a.u. for silicon and boron atoms and 2.5 for potassium. $R_{\mathrm{K}_{\max }}$ was fixed to 4.0 and the geometry optimization was performed first with only the $\Gamma k$ point and then with $40 k$ points in the Brillouin zone. The $\mathrm{K} / \mathrm{Si}(111)$ :B surface was modeled using a supercell made of 8 layers of silicon which were saturated with hydrogen atoms at the lower face. A layer of $\mathrm{Si}$ adatom was then added at the $T_{4}$ position. The six potassium atoms were placed half way between the $\mathrm{Si}$ adatoms occupying the center of the cell and the center of the unit cell lattice vectors and the 4 boron atoms were substituted to Si atoms in the second layer underneath the surface, directly under the adatoms. This site is called $\mathrm{S}_{5}$ in the literature, because it has 5 near neighbors.

The main results concerning structural aspects are summarized in Fig. 1(b). Two distinct Si adatom sites associated with two distinct $\mathrm{B}$ sites in proportion $3 / 4$ and $1 / 4$ are clearly evidenced. On one hand, a giant vertical displacement $\delta z=+0.77 \AA$ is observed for $3 / 4$ of the Si adatom (light blue) as the signature of electron doping due to the K-Si proximity $\left(d_{\mathrm{K}-\mathrm{Si}}=3.38 / 3.61 \AA\right)$. On the other hand, $1 / 4$ of the $\mathrm{Si}$ adatoms remains undisplaced since they are situated far away from the $\mathrm{K}$ adatoms $\left(d_{\mathrm{K}-\mathrm{Si}}=5.11 \AA\right)$. In this later case, the $\mathrm{Si}$ adatom-B bond length of $2.16 \AA$ corresponds to what has been measured and calculated for the $\mathrm{Si}: \mathrm{B}$ substrate $[26,27]$. As a consequence, an alternation of short $(2.16 \AA)$ and long $(2.93 \AA) \mathrm{Si}$ adatom-B bonds or equivalently of elongated and undistorted $\mathrm{Si}$ tetrahedra is observed on the hexagonal lattice leading to the expected $2 \sqrt{3}$ surface reconstruction. Previous ab initio DFT calculations carried out for 0.33 monolayer have predicted a $\mathrm{K}$ adatom adsorption in the $H_{3}$ or $T_{4}^{\prime}$ sites with a net energy gain per $\sqrt{3}$-unit cell being, respectively, $\delta E=-0.97$ or $-0.87 \mathrm{eV}$ [5]. Here, it is interesting to remark that $\mathrm{K}$ adatoms occupy an intermediate position in between these two sites. In addition, the K-K bond length of $4.7 \AA$ is larger than the $4.54 \AA$ expected for a metallic bond in agreement with a charge transfer mainly to the $\mathrm{Si}$ :B substrate. Therefore, the highest binding energy site, 
(a) LAPW-GGA
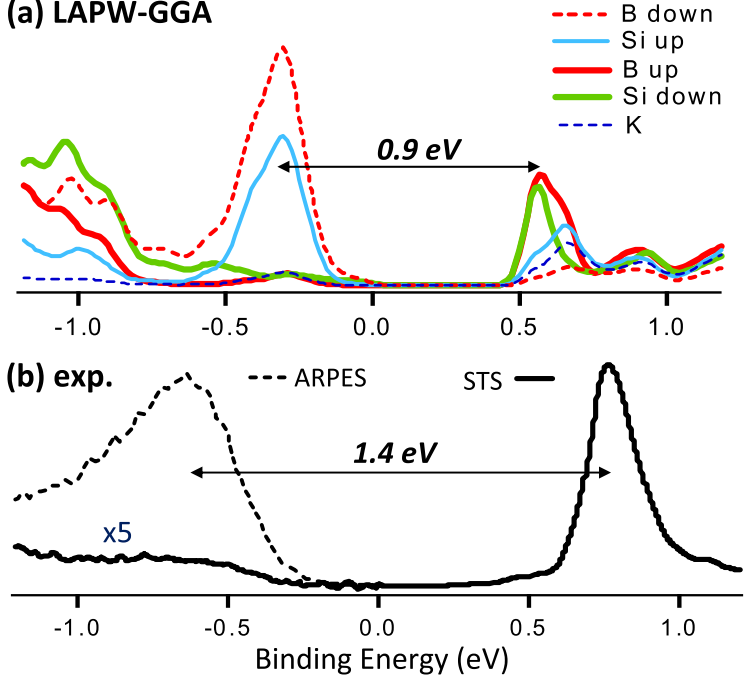

FIG. 2 (color online). (a) Projected surface DOS extracted from calculations; (b) experimental surface band measured by STS at $T=77 \mathrm{~K}$ (black line) and ARPES at $k=K_{2 \sqrt{3}}$ and $T=$ $55 \mathrm{~K}$ (dashed black line).

counting for $1 / 4$ is associated to a short B-Si adatom bond and a long $\mathrm{Si}$ adatom-K bond corresponding to the absence of electron doping in the $\mathrm{Si}$ adatom dangling bond, i.e., an empty dangling bond. At the opposite, the lowest binding energy site, counting for $3 / 4$, is related to the strongly elongated B-Si adatom bond and the shortest $\mathrm{Si}$ adatom-K bond length leading to an effective electron doping of the corresponding $\mathrm{Si}$ adatom dangling bonds. As a consequence, the observation of two distinct $\mathrm{B} 1 s$ peaks in photoemission spectroscopy is a clear signature of the charge transfer associated with the $2 \sqrt{3}$-surface reconstruction. Starting from our structural optimization, B $1 s$ core levels chemical shifts have been calculated by taking into account the optimized lattice potential. As a striking point, two contributions emerged and the calculated chemical shift of $1.09 \mathrm{eV}$ is in good agreement with the experimental one of $1.14 \mathrm{eV}$ validating thus the structural model we proposed.

The projected densities of states (DOS) have been calculated in order to be compared with our ARPES and STS measurements. The results are summarized in Fig. 2. The calculated DOS exhibits two well-defined contributions for occupied and unoccupied states at low energy attributed to the K-induced surface states. Indeed, mainly $\mathrm{Si}, \mathrm{B}$, and to a lesser extent $\mathrm{K}$ surface atoms contribute to the density of states in the energy range $-0.9 \mathrm{eV} /+0.9 \mathrm{eV}$. As expected, the occupied part of the calculated DOS has a $\mathrm{Si}$ up adatom character located at $-0.30 \mathrm{eV}$ but surprisingly even stronger is the B down character. This is consistent with a charge transfer in a strongly hybridized Si adatom-B orbital and is the key point used to understand the extreme sensitivity of $\mathrm{B} 1 s$ core levels to charge ordering. The $\mathrm{K}$-transferred electron is efficiently delocalized along the
$\mathrm{Si}$ adatom-B bond. At the opposite, the unoccupied part of the calculated DOS presents a dominant Si down adatom-B up character located at $+0.57 \mathrm{eV}$. In addition, $\mathrm{K}$ DOS contributes only slightly to the occupied surface band as expected for an electron doping of the Si:B interface. For comparison, the empty states probed by the STS spectrum presented in Fig. 2(b) exhibits a well-defined prominent peak at $0.75 \mathrm{eV}$ isolated from the $\mathrm{K} / \mathrm{Si}$ :B conduction band starting around $1.2 \mathrm{eV}$. At the opposite, the filled states are characterized by a very weak contribution located at $-0.72 \mathrm{eV}$ whose energy is in good agreement with the surface band measured by ARPES. Hence, the two welldefined peaks obtained by combining STS and ARPES data can be qualitatively assigned to the empty ( $\mathrm{Si}$ down-B up) and filled (Si up-B down) calculated surface DOS. Therefore, the insulating character of the $2 \sqrt{3}$-alkalimetal/Si:B can be understood as a consequence of the strong vertical lattice distortion. It should be noticed that the surface gap, defined as the energy difference between the two maxima in the DOS is experimentally larger $(1.4 \mathrm{eV})$ than the calculated one $(0.9 \mathrm{eV})$. This is an usual shortcoming of density functional calculations which can generally be improved, for example, using the $G W$ approximation [30].

Finally, voltage dependent STM images have been calculated within the Tersoff-Haman approximation in the constant current mode. The charge density map is obtained by integrating the energy dependent local density $\rho(\vec{r}, E)$ over the filled and empty part of the surface states as shown in Fig. 2(a). We consider the same filled or empty states as those taken into account when recording STM images at potential $V=-1.2 \mathrm{eV}$ [Fig. 3(c)] and $V=+1.2 \mathrm{eV}$ [Fig. 3(d)] accounting for the smallest calculated gap. The results of the calculations are presented on the inset of Figs. 3(a) and 3(b). Then, a Gaussian convolution has been applied to take into account the finite lateral
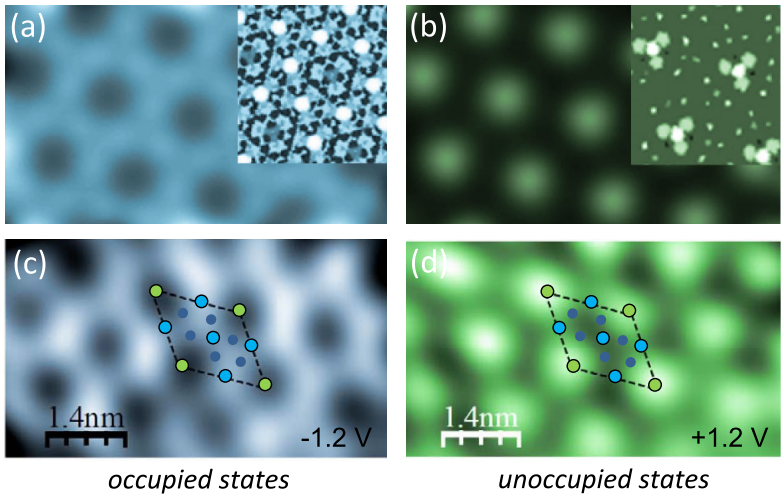

FIG. 3 (color online). Calculated (a),(b) and measured (c), (d) STM images obtained by integrating over occupied (a),(c) and unoccupied (b),(d) surface bands; see explanations in the text; the surface unit cell is shown for clarity, color of atoms are those of Fig. 1. 
resolution leading to simulated STM images presented in Figs. 3(a) and 3(b). On one hand, the calculated filled states image exhibits intensity maxima on Si up adatoms, therefore revealing the Si up-B down bond, whereas intensity goes down to zero on Si down adatoms. On the other hand, the calculated empty states image evidence a drastic inversion contrast with intensity maxima localized on $\mathrm{Si}$ down adatoms. STM measurements presented in Figs. 3(c) and 3(d) evidence clearly this inversion of contrast which can be considered as a signature of the charge redistribution induced by atoms displacements. A $2 \sqrt{3}$ surface unit cell containing Si up (light blue), down (green) and $\mathrm{K}$ (dark blue) adatoms is shown in order to evidence the charge localization. As expected from the calculated density of states, intensity maxima are mainly observed on $\mathrm{Si}$ up adatom for occupied states and on Si down adatom for empty states whereas almost no intensity is observed on $\mathrm{K}$ adatoms confirming that electrons are mainly delocalized in Si-B hybridized dangling bonds. Therefore, a very good agreement is obtained between calculated and measured STM images showing that DFT calculations have captured here the alkali-metal-induced charge density contours. Moreover, to quantify the charge redistribution we have integrated the partial density of states of Fig. 2 from $-0.7 \mathrm{eV}$ to $\mathrm{E}_{F}$. We found 0.1 and 0.19 for Si up and B down atoms and 0.008 and 0.025 for Si down and B up atoms. Hence, a moderate charge modulation $\delta q \approx 0.3 e$ is obtained from DFT calculations being far from the value $\delta q=2 e$ expected in the bipolaronic limit previously supposed to be reached $[18,19]$.

In summary, we proposed an atomic model for the $2 \sqrt{3}$ surface reconstruction of $0.5 \mathrm{ML} \mathrm{K} / \mathrm{Si}(111)$ : B where $3 / 4$ of the $\mathrm{Si}$ adatoms are strongly displaced upward in the $z$ direction. STM images reveal that potassium adatoms lose their electrons in favor of the above mentioned $\mathrm{Si}-\mathrm{B}$ molecular orbital. As a consequence of this charge transfer we observe a shift of the $1 s$ states of boron atoms being directly underneath these $\mathrm{Si}$ adatoms. From this giant $\mathrm{Si}$ adatom displacement, we obtain an alternation of long and short Si-B bond which form, respectively, the valence and conduction surface bands separated by a large gap induced by the lattice distortion. Therefore, the insulating character of alkali-metal/Si:B interfaces has been captured to an excellent accuracy, from the low lying $1 s$ state of boron, to the unoccupied states above the gap, within a one-electron approach without resorting to many-body physics. Such a buckling of $\mathrm{Si}$ adatoms is a common feature of several semiconducting surfaces but is considerably larger than those usually observed. The bipolaronic limit does not seem to be reached here but the physical origin of this singular behavior, i.e., a giant lattice distortion accompanied by a strongly inhomogeneous electron doping of dangling bonds has to be pursued. This behavior should therefore also be our concern in the way of elucidating the ground state of several other closely related systems like the debated family of $(\mathrm{Pb}, \mathrm{Sn}) /(\mathrm{Ge}, \mathrm{Si})-(111)$ surfaces.

This work has received the financial support of the French ANR SURMOTT program (ANR-09-BLAN0210-01) and the Spanish MICIIN under Project No. FIS2010-16046.

[1] H. H. Weitering, X. Shi, P. D. Johnson, J. Chen, N. J. DiNardo, and K. Kempa, Phys. Rev. Lett. 78, 1331 (1997).

[2] C.S. Hellberg and S. C. Erwin, Phys. Rev. Lett. 83, 1003 (1999).

[3] G. Santoro, S. Scandolo, and E. Tosatti, Phys. Rev. B 59, 1891 (1999).

[4] F. Flores, J. Ortega, and R. Perez, Surf. Rev. Lett. 6, 411 (1999).

[5] H. Q. Shi, M. W. Radny, and P. V. Smith, Phys. Rev. B 70, 235325 (2004).

[6] L. I. Johansson, F. Owman, and P. Mårtensson , Surf. Sci. 360, L478 (1996).

[7] N. F. Mott, Proc. R. Soc. A 62, 416 (1949).

[8] J. Hubbard, Proc. R. Soc. A 276, 238 (1963).

[9] R. Cortes, A. Tejeda, J. Lobo, C. Didiot, B. Kierren, D. Malterre, E. G. Michel, and A. Mascaraque, Phys. Rev. Lett. 96, 126103 (2006).

[10] S. Modesti, L. Petaccia, G. Ceballos, I. Vobornik, G. Panaccione, G. Rossi, L. Ottaviano, R. Larciprete, S. Lizzit, and A. Goldoni, Phys. Rev. Lett. 98, 126401 (2007).

[11] V. I. Anisimov, A. E. Bedin, M. A. Korotin, G. Santoro, S. Scandolo, and E. Tosati, Phys. Rev. B 61, 1752 (2000).

[12] A. E. Trumper, C. J. Gazza, and L. O. Manuel, Phys. Rev. B 69, 184407 (2004).

[13] G. Profeta and E. Tosatti, Phys. Rev. Lett. 95, 206801 (2005).

[14] G. Profeta and E. Tosatti, Phys. Rev. Lett. 98, 086401 (2007).

[15] J. Ortega, R. Pérez , and F. Flores, J. Phys. Condens. Matter 14, 5979 (2002).

[16] K. C. Pandey, Phys. Rev. Lett. 49, 223 (1982).

[17] R. M. Feenstra and J. A. Stroscio, Phys. Rev. Lett. 59, 2173 (1987).

[18] L. A. Cardenas, Y. Fagot-Revurat, L. Moreau, B. Kierren, and D. Malterre, Phys. Rev. Lett. 103, 046804 (2009).

[19] C. Tournier-Colletta, L. Cardenas, Y. Fagot-Revurat, B. Kierren, A. Tejeda, D. Malterre, P. le Fevre, F. Bertran, and A. Taleb-Ibrahimi, Phys. Rev. B 82, 165429 (2010).

[20] C. Tournier-Colletta, L. Cardenas, Y. Fagot-Revurat, B. Kierren, A. Tejeda, and D. Malterre (to be published).

[21] A. Tejeda, R. Cortes, J. Lobo-Checa, C. Didiot, B. Kierren, D. Malterre, E. G. Michel, and A. Mascaraque, Phys. Rev. Lett. 100, 026103 (2008).

[22] Y. Ma, J.E. Rowe, E.E. Chaban, C. T. Chen, R. L. Headrick, G. M. Meigs, S. Modesti, and F. Sette, Phys. Rev. Lett. 65, 2173 (1990).

[23] Y. Ma, C. T. Chen, G. M. Meigs, F. Sette, G. Illing, and H. Shigakawa, Phys. Rev. B 45, 5961 (1992). 
[24] T. M. Grehk, P. Martensson, and J. M. Nicholls, Phys. Rev. B 46, 2357 (1992).

[25] T. M. Grehk, L. S. O. Johansson, U. O. Karlsson, and A. S. Flodstrom, Phys. Rev. B 47, 13887 (1993).

[26] P. Baumgartel, J. J. Paggel, M. Hasselblatt, K. Horn, V. Fernandez, O. Schaff, J. H. Weaver, A. M. Bradshaw, D. P. Woodruff, E. Rotenberg, and J. Denlinger, Phys. Rev. B 59, 13014 (1999).
[27] H. Q. Shi, M. W. Radny, and P. V. Smith, Phys. Rev. B 66, 085329 (2002).

[28] J.P. Lewis et al., Phys. Rev. B 64, 195103 (2001).

[29] J. P. Perdew, K. Burke, and M. Ernzerhof, Phys. Rev. Lett. 77, 3865 (1996).

[30] L. Hedin and S. Lundqvist, Solid State Phys. 23, 1 (1970). 